This is the peer reviewed (accepted) version of the following article:

Leugnerova, M., Vaculik, M., \& Prochazka, J. (2016). The Influence of Candidate Social Effectiveness on Assessment Center Performance Ratings: A field study. International Journal of Selection and Assessment, 24(2), 150-160. doi: 10.1111/ijsa.12137

which has been published in final form at https://doi.org/10.1111/ijsa.12137

This article may be used for non-commercial purposes in accordance with Wiley Terms and Conditions for SelfArchiving.

\title{
The influence of candidate social effectiveness on assessment center performance ratings: A field study
}

\author{
Authors \\ Marcela Leugnerova, Martin Vaculik and Jakub Prochazka
}

Institutional Affiliation

Department of Psychology, Faculty of Social Studies, Masaryk University, Brno, Czech Republic

\begin{abstract}
Author Note
Correspondence concerning this article should be addressed to Marcela Leugnerova, Department of Psychology, Faculty of Social Studies, Masaryk University Brno, Jostova 10, 60200 Brno, Czech Republic. Electronic mail may be sent to 333166@mail.muni.cz.
\end{abstract}




\begin{abstract}
The present research examined the influence of constructs representing social effectiveness on assessment center (AC) ratings in two samples. We expected different effects of self-monitoring (SM) on different dimension ratings, a positive effect of the ability to identify criteria (ATIC) on the overall AC rating and a moderating effect of the ATIC on the relationship between SM and the dimension rating. Forty-six (Study 1) and 115 (Study 2) applicants participated in ACs in field settings. Across both studies, SM had a negative effect on the integrity rating. No relationship was identified between SM and social sensitivity or problem solving ratings. In Study 1, the ATIC had a positive effect on the overall AC rating. No support was identified for a moderating effect of the ATIC on the relationship between SM and the social sensitivity rating.
\end{abstract}

Key words: ability to identify criteria, self-monitoring, assessment center, social effectiveness 


\section{Introduction}

Applicant performance in a selection process is influenced by the level of targeted skills and abilities, as well as the effectiveness in reading, understanding and controlling social interactions (Ferris, Perrewé, \& Douglas, 2002). We focused on two important constructs which represent this effectiveness and which are particularly important to perform well. The first is the accuracy with which participants are able to identify what is assessed in the situation at hand (this aspect is best represented by the ability to identify criteria, ATIC; Kleinmann, 1993), the second aspect is the motivation and ability to present themselves in accordance with the perceived requirements of the situation (best represented by self-monitoring, SM; Snyder, 1974).

The current body of research does not clearly indicate how these two constructs jointly influence performance in a selection process. In fact, an applicant may have motivation and ability to adjust his self-presentation (SM) but may not be able to correctly guess what is assessed in the selection process (ATIC). Consequently, he or she may modify his or her self-presentation based on erroneous conclusions regarding the factors assessed. Similar to Klehe et al. (2012), we also suggest that considering both ATIC (as ability to identify what is assessed) and SM (as motivation to tailor self-presentation to demands and ability to do so) together can help us to more accurately understand how effectiveness in reading, understanding and controlling social interactions influences applicant performance in a selection process.

The positive influence of the ATIC on performance has been demonstrated during selection simulations in a sample of students (for review, Kleinmann et al., 2011). The relationship between SM and performance in a selection process has not been clearly supported; the correlations between SM and overall performance in a selection interview range between $r=$ .05 - .10 (Anderson, Silvester, Cunningham-Snell, \& Haddleton, 1999; Cook, Vance, \& Spector, 2000). Partial support for this relationship has been demonstrated by Anderson and Thacker 
(1985) who reported a positive correlation between SM and overall performance of woman in an assessment center (AC; $r=.45$ ). The reason for the differential findings may be explained by the fact that the multidimensionality of performance was not taken into account (Motowidlo, Borman, \& Schmit, 1997); thus, not all dimensions of performance are influenced by SM in the same way. In our research, we therefore focused on the influence of SM on dimension ratings in a selection process, which included influence of the ATIC on an overall rating and influence of both SM and ATIC on dimension ratings in a selection process. All relations were studied in a field setting in two different samples that comprised employees of multinational companies and university students. The rationale is that the majority of studies to date that have focused on the influence of the ATIC on performance in a selection process have been conducted as a selection simulation for university students (e.g., Melchers et al., 2009). However, a selection process in a field setting may differ from a selection simulation in several domains regarding factors such as the level of experienced stress or motivation to succeed. The two groups of candidates may also exhibit several different features, for example, the amount of experience with performance evaluation both in the selection situation and workplace. For this reason, we compared the influence of SM and ATIC in a field setting in these two different samples.

\section{Self-monitoring}

The nature of SM lies in individual differences in motivation and ability to control the images of self, which individuals project in a social interaction (Snyder, 1987). Individuals high in SM guide their behavior in interpersonal situations on the basis of situational considerations and use techniques of impression management (IM) to seek approval from other individuals. In contrast, individuals low in SM are less attentive to social information regarding appropriate behavior, and their self-presentation is controlled by inner attitudes, dispositions, and values 
(Gangestad \& Snyder, 2000).

There are two potential explanations for differences in performance ratings between individuals with different levels of SM. The first explanation is that high and low self-monitors differ in their motivation to use IM strategies in appraisal situations to influence a rater and in their efficiency in doing so. High self-monitors use these strategies more often and more efficiently (Barbuto \& Moss, 2006; Turnley \& Bolino, 2001). Duarte and Goodson (2012) demonstrated that individuals high in SM received a better performance rating compared with individuals low in SM when their performance was deficient or when they perceived themselves to be in an unfavorable relationship with their supervisor. These findings indicate that high selfmonitors may have attempted to compensate for a low objective performance or poor relationship with their supervisors via engagement in appropriate self-presentation behaviors to influence their supervisor's impression of them.

The second explanation for the better performance rating of high self-monitors may be that they have better skills compared with low self-monitors. According to some authors, high self-monitors are more effective in interpersonal relationships because they are more sensitive to various situational demands and choose the correct reactions. Warech, Smither, Reilly, Millsap, and Reilly (1998) studied the underling rationale for the relations between SM and 360-degree feedback. They assumed that if the explanation for better ratting is solely the employment of IM strategies, then high self-monitors would be rated better on all dimensions (interpersonal ${ }^{1}$ and business $^{2}$ ) and only by their supervisors, who are targeted by the IM strategies. However, in the actual study, high self-monitors were rated better only on interpersonal dimensions and by both

\footnotetext{
${ }^{1}$ Interpersonal dimensions, e.g., empowerment, managing teams, influencing other individuals

${ }^{2}$ Business dimensions, e.g., planning, decision making, strategic thinking, business knowledge
} 
supervisors and assessors. Furthermore, in another study, high self-monitors reported a lower frequency of conflict with other individuals and a stronger preference for cooperation in conflict resolution (Baron, 1989). It has not yet been clearly established which of these theories explains why individuals high in SM receive better ratings.

It could be expected that high self-monitors will be strongly motivated to present themselves in the best possible light in a selection process (high-stakes situation). Fandt and Ferris (1990) demonstrated that high self-monitors utilize IM strategies more often than low selfmonitors during a selection process. At the same time (in accordance with the second explanation for the differences in performance ratings between high and low self-monitors), high selfmonitors may exhibit higher levels of abilities on some dimensions. Nevertheless, there is relatively little attention paid to the relation between SM and performance ratings in a selection process, and current research has not provided substantial support for the existence of this relationship (e.g., Cook et al., 2000) or has only supported in part (Anderson \& Thacker, 1985).

Considering the opacity of the relation between SM and performance ratings in a selection process, it is important to determine why this relationship is either not detected or weaker than expected.

\section{Self-monitoring and the multidimensionality of performance}

The reason that researchers were not able to identify a clear relationship between SM and performance may be the lack of consideration of the different dimensions of performance.

Motowidlo et al. (1997) suggested that job performance consists of different behavioral episodes (which support or prevent fulfillment of organizational goals). To determine the episodes that predict performance, performance domains should be organized into behaviorally homogeneous 
categories because a particular variable may only predict some behavioral episodes. Day and Silverman (1989) suggested that the multidimensionality of performance is one potential factor that may have decreased the reported relationship between personality variables (e.g., SM) and job performance. Accordingly, Caligiuri and Day (2000) demonstrated that SM has differential effects across three distinct performance dimensions. Self-monitoring was positively correlated with performance on expatriate-specific dimensions, negatively correlated with contextual performance and unrelated to technical performance. Similarly, Schleicher, Day, Mayes, and Riggio (2002) reported that SM was positively correlated with communication and leadership skills. However, SM was not correlated with decision-making skills. Studies that have particularly focused on performance in a selection process did not provide support that SM relates differently to different performance dimensions (e.g., Anderson et al., 1999; Cook et al., 2000). However, the authors did not define the measured dimensions, which makes what was included in the dimensions unclear and therefore the relations that could be expected. In our research, we hypothesized that individuals with different levels of SM would differ in performance on different dimensions based on their potential to perform better in a particular dimension and the susceptibility of this dimension to be influenced by IM.

We assumed that some dimensions are more susceptible to IM influence compared with other dimensions. This susceptibility to influence is based on their stability. Stable dimensions require substantial effort and time to be changed. Less stable dimensions comprise skills that could be developed as a result of recent educational experiences and are thus more subject to change (Thornton \& Rupp, 2006). It could be expected that the rating of a less stable dimension (e.g., interpersonal) could be more influenced by IM than the rating of a more stable dimension (e.g., cognitive skills). 
We expected that SM would influence performance on the social sensitivity dimension, which is defined as ability to build relationships with other individuals, kindness, thoughtfulness, willingness to offer help, and willingness to make compromises. First, we assumed that this dimension could be considered less stable and thus more susceptible to IM influence. Second, there are differences between high and low self-monitors in interpersonal skills. The basic motivation of high self-monitors is the enhancement of their social status; thus, their behavior is navigated by the receipt or maintenance of approval from other individuals. Ickes, Holloway, Stinson, and Hoodenpyle (2006) reported that individuals high in SM are motivated to induce positive affect in other individuals and express more positive affect in their social selfpresentations. Moreover, high self-monitors were more likely to help co-workers and communicate with them in a way that facilitates individual and group performances (Blakely, Andrews, \& Fuller, 2003).

H1: Applicant SM is positively related to the social sensitivity dimension rating.

Furthermore, we expected that SM would have significantly lower effect on the problem solving dimension performance which is defined as ability to identify the core of the problem, ability to stay on top of things, ability to perceive relations between pieces of information and logical reasoning. We assumed that this dimension is more stable; thus is less susceptible to IM influence. Moreover, no difference has been identified between high and low self-monitors in cognitive ability. In their meta-analytic study, Day, Schleicher, Unckless, and Hiller (2002) concluded that SM is only very weakly correlated $(r=.06)$ with cognitive ability. In a more recent meta-analytic study, Wilmot (2011) did not identify a correlation between SM and general intelligence. 
$\mathrm{H} 2$ : SM has significantly higher effect on the social sensitivity dimension rating than on the problem solving dimension rating.

We also expected that SM would negatively influence performance on the integrity dimension which is defined as ability to act naturally, willingness to talk openly regarding own limitations, willingness to admit a mistake and self-reflection. The behavior of high self-monitors is governed by a self-enhancement motive, which is manifested by the effort to present oneself to other individuals in a flattering manner. Ickes et al. (2006) suggested that in some situations, such as a selection interview, individuals high in SM are so strongly motivated by the desire to evoke a positive affect in other individuals that their very effort to do so might undermine the very same positive moods and impressions that they work hard to obtain. Similarly, when Simpson, Gangestad, and Biek (1993) asked raters to evaluate the videotapes of participants who answered questions posed by an extremely attractive opposite-sex interviewer, they demonstrated that individuals high in SM were rated as less genuine than individuals low in SM. These findings raise the possibility that the affectively positive and sympathetic self-presentations of high selfmonitors may be exaggerated to an extent that they could be perceived as insincere.

H3: Applicant SM is negatively related to the integrity dimension rating.

\section{Ability to identify criteria}

Performance during a selection process can also be influenced by the ability to estimate the factors evaluated in the situation (ATIC). Kleinmann (1993), who coined the term ATIC, assumed that different selection procedures have various degrees of transparency regarding the 
targeted assessment criteria. For example, the purpose of cognitive ability tests is evident, which makes it easier for applicants to identify assessment dimensions (transparent situation; König, Melchers, Kleinmann, Richter, \& Klehe, 2007). The identification of targeted dimensions in other selection procedures is often less straightforward. For example, in a leaderless group discussion, it might not be clear whether the nature of the situation is competitive or cooperative (nontransparent situation). Studies of non-transparent selection methods suggest that there are stable differences among individuals in the ability to discern what is required in the actual assessment situation (Speer, Christiansen, Melchers, König, \& Kleinmann, 2014). The accuracy of targeted dimension identification in assessment situations is positively correlated with the performance rating in a selection interview (Melchers et al., 2009), overall AC rating (König et al., 2007) and integrity test scores (König, Melchers, Kleinmann, Richter, \& Klehe, 2006).

Findings of studies focused on the ATIC are often limited by their research design because the majority of these studies are conducted as a selection simulation for university students (e.g., Melchers et al., 2009). However, a field setting may be different from a simulation in several aspects, e.g., participants may be under more pressure, which could make them pay less attention to what is measured in the situation at hand and thus use their ATIC to a lesser extent. However, participants in a field setting may have a higher motivation to succeed, which may lead them to use the ATIC more and thus exhibit a stronger relationship between their ATIC and performance ratings.

Another limitation of previous studies is the use of samples of university students (e.g., König et al., 2007) who may differ from a real applicant sample (e.g., in the amount of selection experiences). More experience could lead applicants to use their ATIC less. Based on their previous experiences, they could automatically assume what the assessed criteria are and reflect less on whether these criteria hold true in the current selection situation. In our research, we thus 
decided to conduct two studies and compare the influence of the ATIC on performance ratings in non-student and student applicant samples.

H4: Applicant ATIC is positively related to overall selection performance rating.

\section{Relationship between SM and the ATIC}

Self-monitoring and the ATIC are constructs of a different type. ATIC has significant cognitive component that is reflected in a moderate correlation between the ATIC and cognitive ability (Melchers et al., 2009). Self-monitoring represents the motivation and ability to tailor selfpresentation to identified demands, which is connected to the more frequent use of IM strategies (Barbuto \& Moss, 2006). In our study we tested the assumption that SM and the ATIC interplay in the influence of performance. This assumption corresponds with the suggestions of several authors (Jansen, Lievens, \& Kleinmann, 2011) to focus on the relation between the ATIC and other concepts related to social effectiveness.

As we stated earlier, there have not been found any support for SM influencing performance in selection process (e.g. Cook et al., 2000). Except from multidimensionality of performance there could be another explanation - SM influences performance during selection process only under certain conditions and this relationship is moderated by other variables. Lazar, Kravetz and Zinger (2004) point out to this fact, they found out that relation between SM and performance is shown only in the case, when the assessor was a person with high level of extraversion. We assume that SM (as motivation and an ability to adjust to situation demands) would positively influence performance only when a candidate can correctly identify what is assessed in the situation at hand. To get good evaluation in a certain AC dimension, a candidate must correctly estimate the factors evaluated in situation (meaning he/she has high level of 
ATIC) and subsequently use their abilities and tailor their own self-presentation to recognized demands. Furthermore, if an individual erroneously identifies targeted dimensions and modifies his or her self-presentation accordingly, he or she may perform worse.

We hypothesized that the ATIC is a moderator in the relationship between SM and performance. Furthermore, we hypothesized that the ATIC positively influences the performance of high self-monitors and does not influence the performance of low self-monitors. We assumed that this hypothesis would hold true in less stable dimensions, such as social sensitivity.

H5: ATIC moderates the relationship between SM and the social sensitivity dimension rating.

\section{Methods}

\section{Procedure}

Twenty-three ACs were included in a two-year timespan. All ACs had the same design (same dimensions and exercises), and all assessors received the same training.

AC design. Standardized ACs that fully complied with the standards of the Task Force on Assessment Center Guidelines (2009) were used. Five dimensions, including social sensitivity, problem solving, and integrity, were measured in the ACs. The ACs were composed of five exercises, including two leaderless group discussions, negotiation role-play, supervisory roleplay, and interviews. Six to eight assessors divided into groups of 3-4 individuals participated in each AC. At each AC, every applicant was assessed by all assessors. At the end of each AC, the participants were asked to complete a SM scale and ATIC assessment procedure. All participants were informed that the data collection is part of a research, their responses would not be used to make a selection decision and they could withdraw from research participation. 
Assessor training. To develop common expectations of performance and the meaning of the rating scale levels, all assessors received 12 hours of frame-of-reference training (Schleicher et al., 2002). The assessors were trained to focus on the behaviors likely to be displayed via assessments with varying degrees of proficiency on each dimension. They spent a great deal of time discussing the nature of the job and the meaning of the dimensions. The training also included activities in which the assessors practiced assessments and engaged in discussions regarding differences in the ratings assigned by individual assessors. This training was designed to ensure that every observed behavior is recorded and evaluated the same way by each assessor.

\section{Measures}

Self-monitoring. Self-monitoring was measured with the 13-item Revised SelfMonitoring Scale developed by Lennox and Wolfe (1984). In our studies, we used a 5-point scale format with which the participants indicated their agreements with given statements. Day et al. (2002) reported an alpha coefficient of .80, which is similar to the alpha coefficient in our studies $(\alpha=.76$ in Study 1 and $\alpha=.77$ in Study 2$)$.

Ability to identify criteria. To measure participant ATIC, we combined the methods described by Melchers et al. (2009) and Jansen et al. (2011). The participants completed a questionnaire after the last exercise. Questionnaire included two model situations, where participants received the following instructions: "In the previous exercises, you might have thought about what the observers were assessing. What assumptions did you have during the exercise about what the exercise was intended to assess?". The participants could write down three assumptions per exercise, which corresponded to the number of dimensions assessed in each situation. Similar to Melchers et al. (2009), two trained psychologists independently coded 
the participants' assumptions. In the first step, they determined whether an assumption corresponded to one of the three target dimensions (and identified the specific dimension). In the second step, they rated the strength of this correspondence on a 4-point scale. Alternatively, they could also indicate that the assumption did not correspond to any of the dimensions. In the first step, the inter-rater reliability was Cohen's $\kappa=.71(p<.001)$. As for the correspondence ratings from the second step, the intraclass correlation (ICC 2,1) between the two coders was $r=.89$. Both values are similar to the values reported by Melchers et al. (2009). The coefficients of interrater reliability are calculated across both studies.

To determine the ATIC score, a procedure consistent with Jansen et al. (2011) was used. If none of the assumptions were linked to the consensually determined dimensions, a score of 0 was assigned for situation perception. If an assumption was linked to a consensually determined dimension, the coders' ratings of the strength of the fit between their assumption and the dimension was used as the participant's score on the perception of situational demands. The coders rated all answers independently. If the scores were different, they decided on a dimension correspondence and assigned a rating of correspondence based on a discussion. In the case of ties (several assumptions were linked to the same dimension), the highest strength of the fit rating was used. Therefore, the situation perception scores per dimension could range from 0 (no match between the perceived situational demands and targeted dimensions) to 4 (perfect match between the perceived situational demands and targeted dimensions). The situation perception scores per dimension were averaged across the two exercises.

AC performance. Following the completion of the exercises, a dimension rating was conducted for each participant. The raters used the ORCE model (Ballantyne \& Povah, 2004). First, they reviewed the observable behavior of the participant in a given model situation, 
identified behavior regarding the assessed dimension and rated the participant on the dimension on a 5-point scale that ranged from 0 (poor performance) to 4 (excellent performance). The final exercise dimension rating was the result of a group discussion. To test Hypotheses 1, 2, and 5, an average dimension rating across different exercises was used. To test Hypothesis 3, a dimension rating in a particular exercise was used. This exercise was selected because it assessed the ability to behave naturally and openly, which is an aspect of integrity that we hypothesized could be influenced by SM. To test Hypothesis 4, an overall AC rating was used.

\section{Study 1}

\section{Participants}

The sample consisted of 46 participants $\left(80 \%\right.$ men, $\mathrm{M}_{\mathrm{age}}=34$ years, $\left.\mathrm{SD}_{\mathrm{age}}=8.9\right)$ in $\mathrm{ACs}$ for a team leader position in three private multinational corporations in the engineering, building and banking sector. Selection was organized by an HR consulting company, and ACs comprised the third round of the selection process. The candidates were selected for this round based on their CV and a screening interview.

\section{Results}

The descriptive statistics of the Study 1 variables are shown in Table 1 . Three linear regression analyses were conducted to determine the effects of SM and the ATIC on different dimensions ( $\mathrm{H} 1, \mathrm{H} 2, \mathrm{H} 4$, and $\mathrm{H} 5)$ of AC performance. One ordinal regression was performed to test H3. In all models, both SM and the ATIC were used as predictors to estimate the contribution of one (SM or ATIC) while controlling for the contribution of the other variable. Table 2 summarizes the results of three linear regression analyses. The dependent variable in the first regression analysis was the social sensitivity rating. No significant relationship was identified 
between SM and the social sensitivity rating. Hypothesis 1 was thus not supported. To test H2, regression was conducted with the problem solving rating as the dependent variable. Selfmonitoring was not significantly related to the problem solving rating. The strength of the relationship of SM and the problem solving rating was close to zero. To determine different effect of SM on social sensitivity rating and problem solving rating we tested significance of differences between $\beta$ coefficients from first and second regression analysis. The result was not significant $(\mathrm{z}=0.98, \mathrm{p}=0.328)$. Hypothesis 2 was thus not supported.

To test $\mathrm{H} 3$, we conducted an ordinal regression as the integrity rating, which indicates a dependent variable, was measured on the ordinal scale. Self-monitoring and the ATIC together explained $19.2 \%$ (Cox and Snell) and $21.3 \%$ (Nagelkerke) of the variance in the integrity rating. Our model represented the data better than a model with a constant $\left(\chi^{2}(2)=9.58, p<.05\right)$. In support of Hypothesis 3, an increase in SM was related to a lower integrity rating, as demonstrated by a significant Wald test $(p<.05)$.

As shown in Table 2, the ATIC was a significant predictor of the overall AC rating while controlling for SM. The relationship between the ATIC and overall AC rating was moderately strong. The ATIC was simultaneously a significant predictor of the social sensitivity and problem solving ratings. Our hypothesis $(\mathrm{H} 4)$ that the degree to which applicants are able to identify targeted dimensions is positively related to their performance was supported.

Finally, we hypothesized (H5) that the ATIC is a moderator of the relationship between SM and the social sensitivity rating. This hypothesis was not supported by our data (Table 2); there was no significant interaction between SM and the ATIC in the prediction of the social sensitivity rating. 


\section{Study 2}

\section{Participants}

The sample consisted of 115 participants $\left(57 \%\right.$ men, $\mathrm{M}_{\mathrm{age}}=22$ years, $\left.\mathrm{SD}_{\mathrm{age}}=1.5\right)$ in $\mathrm{ACs}$ for an unpaid team leader position in a voluntary student project referred to as Muniss. The project was organized by the Brno city municipality and three Czech universities and it was designed as a competition (best teams received financial bonus). Study participants were college students, who themselves enrolled on the project and who were interested applied for the team leader position. Team leaders were responsible for leading and coordinating project teams that solved tasks provided by project organizers for half of one year. Their role was to organize meetings, give jobs to other team members, control their work and communicate with the project owners. The selection was organized by the same HR consulting company as in Study 1 . In the last two years there were being held 18 ACs with the same design as described in the Study 1. There were used the same methods administrated in the same way as in the Study 1. The ACs comprised the second round of the selection process. The candidates were selected for this round based on their motivation, which was assessed based on letter of motivation and CV. Ten out of 125 applicants were excluded during the pre-selection process. The reasons to their exclusion were either the fact they had not have provided enough reason to why they wanted to join the project or they had not sent the letter of motivation. The process of pre-selection was nearly identical to a real life selection procedure (as in the Study 1).

\section{Results}

The descriptive statistics of the Study 2 variables are shown in Table 3. Similar to Study 1, three linear and one ordinal regression analyses were conducted to determine the effects of SM and the ATIC on different dimensions of AC performance ratings. Both SM and the ATIC were 
included as predictors in all models to determine the effect of each predictor while controlling for the other predictors. The results of the linear regression analyses are summarized in Table 4 . The social sensitivity rating was a dependent variable in the first regression analysis. No significant relationship was identified between SM and the social sensitivity rating. Thus, H1 was not supported. To test $\mathrm{H} 2$, regression was conducted with the problem solving rating as the dependent variable. Self-monitoring was not significantly related to the problem solving rating. The strength of the relationship of SM and the problem solving rating was close to zero. To determine different effect of SM on social sensitivity rating and problem solving rating we tested significance of difference between $\beta$ coefficients from first and second regression analysis. The result was not significant $(z=0.40, p=0.688)$. Similar to Study 1 , Hypothesis 2 was not supported.

To test $\mathrm{H} 3$, we conducted an ordinal regression as the integrity rating, which indicates a dependent variable, was measured on the ordinal scale. Self-monitoring and the ATIC together explained $6.2 \%$ (Cox and Snell) and 6.8\% (Nagelkerke), respectively, of the variance in the integrity rating. Our model represented the data better than a model with only a constant $(\chi 2(2)=$ $6.77, p<.05)$. In support of Hypothesis 3, an increase in SM was related to a lower integrity rating, as demonstrated by a significant Wald test $(p<.05)$.

In contrast to $\mathrm{H} 4$ and the results of Study 1, the ATIC was not a significant predictor of the overall AC rating, and the relationship between the ATIC and the overall AC rating was weak. Our hypothesis that the degree to which applicants are able to identify targeted dimensions is positively related to their performance was not replicated in Study 2.

Similarly to Study 1, there was no significant interaction between SM and the ATIC in the prediction of the social sensitivity rating. Our hypothesis that the ATIC is a moderator of the 
relationship between SM and the social sensitivity rating was thus not supported.

\section{Discussion}

First, we examined the possibility that SM has differential effects across three distinct performance dimensions (social sensitivity, problem solving, and integrity). In both studies, we demonstrated that SM was negatively related to integrity and had no relation to problem solving or social sensitivity. Our findings support, in part, the findings of previous studies that suggested the possibility of differential personality effects across performance dimensions (Caligiuri \& Day, 2000). Together with the present results, these findings indicate that better prediction can be accomplished by theoretically linking personality variables with relevant performance dimensions.

Integrity. Across both studies, SM negatively influences performance on the integrity dimension. The higher the SM of the participants, the less their behavior was perceived as natural and the less they were willing to talk openly regarding themselves and their own limitations. This finding could be interpreted as high self-monitors vigorously attempt to present themselves in the best light possible, which is perceived as unnatural and false in accordance with the findings of Simpson et al. (1993). Similarly, the inability to admit one's mistake or limitation is consistent with a study by Kilduff and Day (1994). The authors demonstrated that in the case of failure, high self-monitors have a tendency to present only information that would not harm them and their status in the eyes of other individuals. Our findings support the assumption that the good selfpresentation of high self-monitors does not have to be an advantage in a selection process and can be a reason that high SM is not related to good performance in a selection process (e.g., Cook et al., 2000). It is possible that in previous research, the assessment of openness and natural behavior were part of the overall performance in a selection process. Although high self-monitors 
could have been rated worse in these variables, this relation was not supported in the overall performance. Therefore, we suggest it is useful to include an assessment of more dimensions, as well as base a selection decision on performances in individual dimensions rather than the overall performance.

Social sensitivity. Self-monitoring did not influence performance on the social sensitivity dimension. One potential explanation may be that the relation between SM and performance is not linear. As suggested in a study by Barrick, Parks, and Mount (2005), SM can moderate the relationship between personal characteristics and behavior. In particular, SM weakens the link between the level of a personal trait (i.e., the tendency to engage in a certain behavior) and the ability to display this potential when the personality trait level is high. In contrast, SM increases the relationship between a personality trait and behavior in individuals with a low level of the personality trait. In their study, the best performance was achieved by low self-monitors who scored high in personality traits such as extraversion. However, high self-monitors performed better than low self-monitors when they scored low on extraversion. It could be assumed that SM can function as a compensation for individuals who have a lower potential to perform well on certain dimensions. However, individuals with a high potential to perform well may be hindered by a high SM. Therefore, it is possible that the relationship between SM and performance on the social sensitivity dimension was not identified in our research because its influence depends on the potential of individuals to perform well.

An alternative explanation for the finding that high self-monitors did not receive a higher rating is that the social sensitivity dimension used in our research does not adequately tap into the content of SM (Day \& Silverman, 1989). Thus, we suggest the relations between SM and other dimensions of interpersonal skills (e.g., assertiveness) should be investigated. 
Problem solving. Our assumption that influence of SM on social sensitivity dimension would be significantly higher than on problem solving dimension was not confirmed. The reason for that might be that the expected relation between SM and social sensitivity rating was not proved. In both studies the strength of the relationship of SM and the problem solving rating was close to zero which is in contrast to the relations of SM and other dimensions. This finding is in compliance with results of those studies, where they proved that SM has no relation with variables representing the ability to solve problems (e.g. decision making in Schleicher et al., 2002). One potential explanation is that the problem solving rating is not susceptible to IM strategies. According to previous findings (Schleicher et al., 2002), problem solving has been demonstrated to be a more stable dimension; thus, it might have been difficult for high selfmonitors to present themselves in the way that they are perceived as "clever" and thus receive a better performance rating in this dimension. As demonstrated in studies that have focused on the relationship between SM and cognitive abilities (Day et al., 2002), there are no differences in cognitive abilities among individuals with different levels of SM, which may represent an alternative interpretation of our findings.

Second, the results of Study 1 indicate that applicant ATIC positively influences their overall AC performance rating. Individuals who were better at guessing what was assessed in the situation had a higher overall AC performance rating compared with individuals who were worse at such guesses. Our findings broaden the validity of the results obtained by Speer et al. (2014) via the demonstration of their applicability in a field setting and non-student applicants. With this in mind, we can consider the ATIC an important social skill, which can be used to predict performance during the selection process, as well as work performance. In Study 2 (with a student sample in a real selection), the influence of the ATIC on the overall AC performance rating was not supported. The first reason for these divergent findings may be the differences 
between applicants in Studies 1 and 2 in their motivation to succeed in the selection process. In Study 1, the applicants competed for a real job position and completed several rounds of the selection process. In Study 2, the applicants competed for an unpaid position in a project in which they would lead other students over a half-year period. It could be assumed that the motivation was higher in the case of the real job position candidates. A higher motivation to succeed could lead to a higher motivation to use the ATIC and behave in accordance with what is assessed in the situation. Another reason for the dissonant findings in Studies 1 and 2 might be the amount of applicant experience with selection processes. It could be assumed that if an individual had more opportunities to use the ATIC, he or she would be better equipped to take advantage of the identified criteria. If an individual attended several selection processes, he or she might have trained how to behave in accordance with the identified criteria. Our findings suggest that the influence of the ATIC on the AC performance rating could differ for various populations. We suggest the replication of our research in a different sample of applicants for a job position in a field setting. We would also suggest taking into account the amount of experience that applicants have with a selection process, as well as the motivation of applicants to succeed in a selection process.

Third, we examined the possibility that the ATIC is a moderator of the relationship between SM and performance in some dimensions. This hypothesis was not supported. One potential explanation may be the nature of the SM concept, which refers to an individual's effort to look for clues in the behavior of other individuals while interacting with them (Snyder, 1987). In contrast, the ATIC primarily refers to the identification of the criteria assessed in a selection process (Kleinmann, 1993). A question remains as to whether individuals navigate their behaviors in a selection process solely based on what they think is measured or if they also pay attention to the behaviors of other individuals in the situation. For example, an individual 
identifies influencing other individuals as the targeted dimension; however, he or she does not behave assertively because other individuals in the group behave calmly and comply with each other's wishes. In future research, we suggest focusing on the identification of what navigates an applicant's behavior when he or she identifies a targeted dimension yet other individuals in the group behave differently from what is assessed. Does he or she modify his or her behavior to the identified criteria (ATIC prevails) or other applicants (SM prevails)? Furthermore, what determines whether an individual tunes up to what they think is assessed or to behaviors of other applicants in the selection process? In future research, we suggest focusing on the influence of group processes (e.g., informative or normative social influences) or personality variables (e.g., self-esteem, conformity tendency).

\section{Limitations and Practical Implications}

Some limitations of our studies should be noted. First, we used an ATIC scoring procedure in which applicant hypotheses are coded by external coders. One potential weakness of this procedure is that the idiosyncrasies of the participant's hypotheses might not be fully captured by external coding. Previous studies that used a procedure in which the participants determine the correspondence between their hypotheses and targeted dimensions have mostly comprised selection simulations for training purposes. This approach enabled researchers to modify the setting to a greater extent. However, this approach was less attainable in our studies in a field setting. Thus, we utilized a less demanding procedure with externally coded hypotheses. In a comparison of these two procedures, Melchers et al. (2009) reported higher reliability for the procedure in which participants determine the correspondence between their hypotheses and targeted dimensions compared with the procedure with external coders. However, a sufficient reliability of the latter procedure was also supported as the influence of the ATIC on performance 
was identified using both coding procedures. We would suggest the verification of our research findings using the ATIC scoring based on candidate self-coded hypotheses.

Our findings regarding the influence of the ATIC on the AC performance ratings in a field setting suggest that thinking about targeted criteria and the ability to guess them correctly could represent characteristics that predict performance in a selection process. It has also been demonstrated that the ATIC assessed using one selection method predicts performance in other selection methods (König et al., 2007), and the ATIC also predicts job performance (Jansen et al., 2013). In the context of these studies, our research supports the assumption that the ATIC represents an important variable to assess in a selection process. It can reflect a social skill that is also important for job performance; in the workplace, it is equally important to recognize the behaviors required in various situations. However, it is important to verify that the same logic of the influence of the ATIC on performance holds true even when its rating is part of the assessment in a selection process. In the course of previous studies, applicants were told that the results of the method will only be used for research purposes. Further research is needed to establish whether applicants would provide the same answers if the results were used for selection decisions.

Another practical implication originates from our finding regarding the influence of SM on integrity (natural behavior). Based on our findings, we would suggest applicants in a selection process should behave naturally and refrain from attempting to be viewed in a better light because these effort may decrease their performance in a selection process. Behaving naturally and being open may represent assessed criteria albeit not explicitly defined. Not behaving naturally may result in a lower performance rating in a selection process. Furthermore, our findings suggest that it is important to include an integrity dimension in the selection process because it allows trained raters to recognize candidates' stylization. 
Social effectiveness and AC performance ratings

\section{References}

Anderson, L. R., \& Thacker, J. (1985). Self-monitoring and sex as related to assessment center ratings and job performance. Basic and Applied Social Psychology, 6, 345-361. doi: 10.1207/s15324834basp0604_5.

Anderson, N., Silvester, J., Cunningham-Snell, N., \& Haddleton, E. (1999). Relationship between candidate self-monitoring, perceived personality, and selection interview outcomes. Human Relations, 52, 1115-1131. doi: 10.1023/A:1016939809734.

Ballantyne, I., \& Povah, N. (2004). Assessment and development centres. Gower Publishing, Ltd.

Barbuto, J. E., \& Moss, J. A. (2006). Dispositional effects in intra-organizational influence tactics: A meta-analytic review. Journal of Leadership and Organizational Studies, 12(3), 30-52. doi: 10.1177/107179190601200303.

Baron, R. A. (1989). Personality and organizational conflict: Effects of the type A behavior pattern and self-monitoring. Organizational Behavior and Human Decision Processes, 44, 281-296. doi: 10.1016/0749-5978(89)90028-9.

Barrick, M. R., Parks, L., \& Mount, M. K. (2005). Self-monitoring as a moderator of the relationship between personality traits and performance. Personnel Psychology, 58, 745767. doi: 10.1111/j.1744-6570.2005.00716.x.

Blakely, G. L., Andrews, M. C., \& Fuller, J. (2003). Are chameleons good citizens? A longitudinal study of the relationship between self-monitoring and organizational citizenship behavior. Journal of Business and Psychology, 18, 131-144. doi: 10.1023/A:1027388729390. 
Caligiuri, P. M., \& Day, D. V. (2000). Effects of self-monitoring on technical, contextual, and assignment-specific performance: A study of cross-national work performance ratings. Group and Organization Management, 25, 154-174. doi: 10.1177/1059601100252004.

Cook, K. W., Vance, C. A., \& Spector, P. E. (2000). The relation of candidate personality with selection-interview outcomes. Journal of Applied Social Psychology, 30, 867-885. doi: 10.1111/j.1559-1816.2000.tb02828.x.

Day, D. V., Schleicher, D. J., Unckless, A. L., \& Hiller, N. J. (2002). Self-monitoring personality at work: A meta-analytic investigation of construct validity. Journal of Applied Psychology, 87, 390-401. doi: 10.1037//0021-9010.87.2.390.

Day, D. V., \& Silverman, S. B. (1989). Personality and job performance: Evidence of incremental validity. Personnel Psychology, 42, 25-46. doi: 10.1111/j.17446570.1989.tb01549.x.

Duarte, N. T., \& Goodson, J. R. (2012). The compensatory role of self-monitoring in performance appraisal. International Journal of Business and Social Science, 3(24), 1-11. http://ijbssnet.com/journals/Vol_3_No_24_Special_Issue_December_2012/1.pdf

Fandt, P. M., \& Ferris, G. R. (1990). The management of information and impressions: When employees behave opportunistically. Organizational Behavior and Human Decision Processes, 45, 140-158. doi: 10.1016/0749-5978(90)90008-W.

Ferris, G. R., Perrewé, P. L., \& Douglas, C. (2002). Social effectiveness in organizations: Construct validity and research directions. Journal of Leadership and Organizational Studies, 9(1), 49-63. doi: 10.1177/107179190200900104.

Fox, S., \& Spector, P. E. (2000). Relations of emotion intelligence, practical intelligence, general intelligence, and trait affectivity with interview outcomes: It’s not all just „G“. Journal of 
Organizational Behaviour, 21, 203-220. doi: 10.1002/(SICI)10991379(200003)21:2<203::AID-JOB38>3.0.CO;2-Z.

Gangestad, S., \& Snyder, M. (2000). Self-monitoring: Appraisal and reappraisal. Psychological Bulletin, 126, 530-555. doi: 10.1037//0033-2909.126.4.530.

Ickes, W., Holloway, R., Stinson, L. L., \& Hoodenpyle, T. G. (2006). Self-monitoring in social interaction: The centrality of self-affect. Journal of Personality, 74, 659-684. doi: 0.1111/j.1467-6494.2006.00388.x.

International Task Force on Assessment Center Guidelines (2009). Guidelines and ethical considerations for assessment center operation. International Journal of Selection and Assessment, 17, 243-253. doi: 10.1111/j.1468-2389.2009.00467.x.

Jansen, A., Lievens, F., \& Kleinmann, M. (2011). Do individual differences in perceiving situational demands moderate the relationship between personality and assessment center dimension ratings? Human performance, 24, 231-250. doi:

10.1080/08959285.2011.580805.

Jansen, A., Melchers, K. G., Lievens, F., Kleinmann, M., Brändli, M., Fraefel, L., \& König, C. J. (2013). Situation assessment as an ignored factor in the behavioral consistency paradigm underlying the validity of personnel selection procedures. Journal of Applied Psychology, 98, 326-341. doi: 10.1037/a0031257.

Kilduff, M., \& Day, D. V. (1994). Do chameleons get ahead? The effect of self-monitoring on managerial careers. Academy of Management Journal, 37, 1047-1060. doi: $10.2307 / 256612$.

Klehe, U.-C., Kleinmann, M., Hartstein, T., Melchers, K. G., König, C. J., Heslin, P. A., ... , \& Lievens, F. (2012). Responding to a personality tests in selection context: The role of the 
ideal-employee factor. Human Performance, 25, 273-302. doi:

10.1080/08959285.2012.703733.

Kleinmann, M. (1993). Are rating dimensions in assessment centers transparent for participants? Consequences for criterion and construct validity. Journal of Applied Psychology, 78, 988-993. doi: 10.1037/0021-9010.78.6.988.

Kleinmann, M., Ingold, P. V., Lievens, F., Jansen, A., Melchers, K. G., \& König, C. J. (2011). A different look at why selection procedures work: The role of candidates ' ability to identify criteria. Organizational Psychology Review, 1, 128-146. doi:

$10.1177 / 2041386610387000$.

König, C. J., Melchers, K. G., Kleinmann, M., Richter, G. M., \& Klehe, U-Ch. (2006). The relationship between the ability to identify evaluation criteria and integrity test scores. Psychology Science, 48, 369-377. http://www.pabst-publishers.de/psychology-science/32006/ps_3_2006_369-377.pdf

König, C. J., Melchers, K. G., Kleinmann, M., Richter, G. M., \& Klehe, U-Ch. (2007).

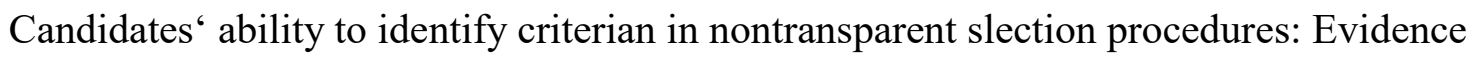
from an assessment center and a structured interview. International Journal of Selection and Assessment, 15, 283-292. doi: 10.1111/j.1468-2389.2007.00388.x.

Lazar, A., Kravetz, S., \& Zinger, A. (2004). Moderating effects of rater personality on the relation between candidate self-monitoring and selection interview ratings. International Journal of Selection and Assessment, 12(4), 321-326. doi: 10.1111/j.0965075X.2004.00287.x.

Lennox, R., \& Wolfe, R. (1984). Revision of the self-monitoring scale. Journal of Personality and Social Psychology, 46, 1349-1364. doi: 10.1037/0022-3514.46.6.1349. 
Melchers, K. G., Klehe, U-Ch., Richter, G. M., Kleinmann, M., König,C. J., \& Lievens, F. (2009). „I know what you want to know“: The impact of interviewees“ ability to identify criteria on interview performance and construct-related validity. Human Performance, 22, 355-374. doi: 10.1080/08959280903120295.

Motowidlo, S. J., Borman, W. C., \& Schmit, M. J. (1997). A theory of individual differences in task and contextual performance. Human Performance, 10, 71-83. doi:

10.1207/s15327043hup1002_1.

Schleicher, D. J., Day, D. V., Mayes, B. T., \& Riggio, R. E. (2002). A new frame for frame-ofreference training. Journal of Applied Psychology, 87, 735-746. doi: 10.1037/00219010.87 .4 .735 .

Simpson, J. A., Gangestad, S. W., \& Biek, M. (1993). Personality and nonverbal social behavior: An ethological perspective of relationship initiation. Journal of Experimental Social Psychology, 29, 434-461. doi: 10.1006/jesp.1993.1020.

Snyder, M. (1974). Self-monitoring of expressive behaviour. Journal of Personality and Social Psychology, 30, 526-537. doi: 10.1037/h0037039.

Snyder, M. (1987). Public appearances, private realities: The psychology of self-monitoring. New York: W. H. Freeman and company.

Speer, A. B., Christiansen, N. D., Melchers, K. G., König, C. J. \& Kleinmann, M. (2014). Establishing the Cross-Situational Convergence of the Ability to Identify Criteria: Consistency and Prediction Across Similar and Dissimilar Assessment Center Exercises. Human Performance, 27, 44-60. doi: 10.1080/08959285.2013.854364.

Thornton, G., \& Rupp, D. (2006). Assessment Centers in Human Resource Management: Strategies for Prediction, Diagnosis, and Development. Lawrence Erlbaum Associates. 
Mahwah, NJ.

Turnley, M. A., \& Bolino, (2001). Achieving desired images while avoiding undesired images: Exploring the role of self-monitoring in impression management. Journal of Applied Psychology, 86, 351-360. doi: 10.1037//0021-9010.86.2.351.

Warech, M. A., Smither, J. W., Reilly, R. R., Millsap, R. E., \& Reilly, S. P. (1998). Selfmonitoring and 360-degree ratings. Leadership Quarterly, 9, 449-473. doi: 10.1016/S1048-9843(98)90011-X.

Wilmot, M. P. (2011). Self-monitoring personality at work revisted: A comparative meta-analysis (Unpublished doctoral dissertation). Retrieved from DigitalCommons at the University of Nebraska - Lincoln. 


\section{Social effectiveness and AC performance ratings}

TABLE 1.

Descriptive statistics and intercorrelations of the variables (Study 1)

\begin{tabular}{|c|c|c|c|c|c|c|c|c|}
\hline Measure & $M$ & $S D$ & 1. & 2. & 3. & 4. & 5. & 6. \\
\hline 1. ATIC & 1.76 & .64 & .47 & & & & & \\
\hline 2. SM & 3.47 & .52 & .11 & .76 & & & & \\
\hline 3. Social Sensitivity & 1.76 & .43 & .27 & .20 & .35 & & & \\
\hline 4. Problem Solving & 1.54 & .44 & $.42 * *$ & -.01 & $.32 *$ & .50 & & \\
\hline 5. Integrity & 2.07 & .77 & .22 & $-.25^{*}$ & .11 & .24 & & \\
\hline 6. Overall Rating & 1.69 & .39 & $.42 * *$ & .19 & $.36^{*}$ & $.66 * * *$ & $.42 * * *$ & .84 \\
\hline
\end{tabular}

Note. $N=46$. Pearson's correlation coefficient was used for all variables with the exception of integrity (ordinal variable), in which Kendall's tau-b coefficient was used. SM = self-monitoring. ATIC = ability to identify criteria.

$* p<.05 . * * p<.01 . * * * p<.001$. 
Social effectiveness and AC performance ratings

TABLE 2.

Results of the regression analyses of the AC ratings on SM and the ATIC

\begin{tabular}{|c|c|c|c|c|c|c|}
\hline Criterion & Predictor & $B$ & $\begin{array}{c}\text { 95\% Confidence } \\
\text { Interval }\end{array}$ & $\beta$ & $p$ & $\Delta R^{2}$ \\
\hline \multirow[t]{6}{*}{ Social sensitivity } & Step 1 & & & & & .13 \\
\hline & SM & .18 & {$[-.09, .46]$} & .19 & .188 & \\
\hline & ATIC & .20 & {$[.04, .40]$} & .30 & .046 & \\
\hline & Step 2 & & & & & .00 \\
\hline & SM x ATIC & -.01 & {$[-.48, .45]$} & -.07 & .957 & \\
\hline & Total $R^{2}$ & .13 & & & & \\
\hline \multirow[t]{3}{*}{ Problem solving } & SM & -.00 & {$[-.28, .27]$} & -.00 & .972 & \\
\hline & ATIC & .28 & {$[.09, .48]$} & .41 & .006 & \\
\hline & $R^{2}$ & .17 & & & & \\
\hline \multirow[t]{3}{*}{ Overall rating } & SM & .16 & {$[-.08, .40]$} & .18 & .189 & \\
\hline & ATIC & .26 & {$[.09, .43]$} & .42 & .003 & \\
\hline & $R^{2}$ & .22 & & & & \\
\hline
\end{tabular}

Note. $N=46 . \mathrm{SM}=$ self-monitoring. ATIC $=$ ability to identify criteria. 


\section{Social effectiveness and AC performance ratings}

TABLE 3.

Descriptive statistics and intercorrelations of the variables (Study 2)

\begin{tabular}{|c|c|c|c|c|c|c|c|c|}
\hline Measure & $M$ & $S D$ & 1. & 2. & 3. & 4. & 5. & 6. \\
\hline 1. ATIC & 2.04 & .59 & .58 & & & & & \\
\hline 2. SM & 3.64 & .46 & $.25 * *$ & .77 & & & & \\
\hline 3. Social Sensitivity & 1.85 & .59 & .04 & -.07 & .54 & & & \\
\hline 4. Problem Solving & 1.90 & .54 & .05 & -.11 & $.33 * *$ & .45 & & \\
\hline 5. Integrity & 2.19 & .84 & .03 & $-.14 *$ & $.31 * *$ & $.29 * *$ & & \\
\hline 6. Overall Rating & 2.00 & .34 & .15 & -.03 & $.46^{* *}$ & $.68 * *$ & $.35^{* *}$ & .72 \\
\hline
\end{tabular}

Note. $N=115$. Pearson's correlation coefficient was used for all variables with the exception of integrity (ordinal variable), in which Kendall's tau-b coefficient was used. SM = self-monitoring. ATIC $=$ ability to identify criteria.

$* p<.05 . * * p<.01$. 
Social effectiveness and AC performance ratings

TABLE 4.

Results of the regression analyses of the AC ratings on SM and the ATIC

\begin{tabular}{|c|c|c|c|c|c|c|}
\hline Criterion & Predictor & $B$ & $\begin{array}{c}\text { 95\% Confidence } \\
\text { Interval }\end{array}$ & $\beta$ & $p$ & $\Delta R^{2}$ \\
\hline \multirow[t]{6}{*}{ Social sensitivity } & Step 1 & & & & & .01 \\
\hline & SM & -.12 & {$[-.38, .13]$} & -.10 & .346 & \\
\hline & ATIC & .06 & {$[-.14, .27]$} & .06 & .533 & \\
\hline & Step 2 & & & & & .00 \\
\hline & SM x ATIC & .04 & {$[-.42, .50]$} & .17 & .866 & \\
\hline & Total $R^{2}$ & .01 & & & & \\
\hline \multirow[t]{3}{*}{ Problem solving } & SM & -.18 & {$[-.40, .05]$} & -.16 & .125 & \\
\hline & ATIC & .08 & {$[-.10, .26]$} & .09 & .370 & \\
\hline & $R^{2}$ & .03 & & & & \\
\hline \multirow[t]{3}{*}{ Overall rating } & SM & -.62 & {$[-.21, .08]$} & -.09 & .393 & \\
\hline & ATIC & .10 & {$[-.02, .21]$} & .17 & .099 & \\
\hline & $R^{2}$ & .03 & & & & \\
\hline
\end{tabular}

Note. $N=115 . \mathrm{SM}=$ self-monitoring. ATIC $=$ ability to identify criteria. 